\title{
SENSEFUL: an SDN-based Joint Access and Backhaul Coordination for Dense Wi-Fi Small Cells
}

\author{
Eduard Garcia-Villegas*, David Sesto-Castilla*, Sven Zehl ${ }^{\dagger}$, Anatolij Zubow ${ }^{\dagger}$, \\ August Betzler ${ }^{\ddagger}$ and Daniel Camps-Mur ${ }^{\ddagger}$ \\ Universitat Politècnica de Catalunya \\ $\dagger$ Technische Universität Berlin \\ \{eduardg, dsesto\}@entel.upc.edu \\ \{anatolij.zubow, zehl\}@ @u-berlin.de \\ $\ddagger$ i2CAT Foundation, Spain \\ \{daniel.camps, august.beztler\}@i2cat.net
}

\begin{abstract}
Dense Small Cell networks are considered the most effective way to cope with the exponential increase in mobile traffic demand expected for the upcoming years and are one of the foundations of the future 5G. However, novel architectures are required to enable cost-efficient deployments of very dense outdoor Small Cell networks, complementing the coverage layer provided by macro-cells. In this regard, two important challenges need to be solved to make this vision a reality: i) increased traffic dynamics, which are translated into more frequent handovers, and ii) cost-efficient deployment of large number of Small Cells. In this paper we propose and evaluate SENSEFUL, an novel architecture addressing the two problems highlighted above: Software-Defined Networking (SDN) as the key technology to promote adaptability to a varying environment and provide efficient mobility solutions in the dense access layer, and novel wireless backhauling technologies where traditional wired connectivity does not meet cost/efficiency restrictions.
\end{abstract}

Index terms- Wi-Fi, SDN, 5G, Small Cells, Architecture

\section{INTRODUCTION}

The deployment of the so called small cells seem to be the architecture towards which wireless access network providers will have to be moving in order to accommodate the eightfold global mobile data traffic increase expected between 2015 and 2020 (according to the most recent Cisco study [1]), year in which the first $5 \mathrm{G}$ networks are expected to be a reality. However, a massive deployment of Small Cells poses significant technical hurdles to current network architectures.

The Small Cell Forum [2] is the organization in charge of supporting the wide-scale adoption of small cells by means of defining standards and promoting their use. In that context, the accepted definition of small cell is "an umbrella term for operator-controlled, low-powered radio access nodes, including those that operate in licensed spectrum and unlicensed carrier-grade Wi-Fi". In fact, the concept of small cell is simply used to define all possible implementations of femtocells, trying to eradicate the idea that femtocells are only used in residential spaces. In particular, for dense outdoor scenarios, high mobility [3] within small-sized cells (frequent handovers), and efficient backhauling (wired connectivity often not feasible), constitute some of the main problems that require innovative solutions, as part of future 5G.

Although future dense Small Cell deployments are likely going to be composed of a set of heterogeneous technologies such as 3GPP LTE, along with its 5G evolutions, and new WiFi technologies (e.g. IEEE 802.11ax, 802.11ay), it is true that nowadays, operator-run Wi-Fi small cells are not as popular as other licensed spectrum-based technologies. One of the main reasons is the use of an unlicensed (and crowded) spectrum by $\mathrm{Wi}-\mathrm{Fi}$, which hampers the efficient and effective use of the medium. This means Wi-Fi operators have to compete for radio resources not only with other Wi-Fi networks, but also with other technologies (mostly in the $2.4 \mathrm{GHz}$ ISM band, but soon also in the $5 \mathrm{GHz}$ band against LTE-U/LAA). What is more, the use of $\mathrm{Wi}-\mathrm{Fi}$ transmissions also in a wireless backhaul further aggravates radio resource management issues or, in other words, makes the management of small cell infrastructure an even more interesting challenge.

With the introduction of novel techniques such as the ones detailed in the following sections, we argue that Wi-Fi based small cells can become a reality. Hence, our main goal in this work is to propose novel network architectures for future dense outdoor small cell networks using Wi-Fi in order to enable seamless mobility and an efficient wireless self-backhauling. More precisely, in this paper we present SENSEFUL, a novel architecture for SDN-based joint access and backhaul coordination for Wi-Fi small cells to tackle the aforementioned problems of mobility and wireless backhauling.

The remaining of this paper is structured as follows. Section II details the proposed architecture for SENSEFUL; then, the evaluation scenario is described in III and the results obtained thereof are discussed in section IV. Finally, some concluding remarks are given in $\mathrm{V}$.

\section{THE SENSEFUL ARCHITECTURE}

The architecture proposed in this paper consists of hybrid backhaul (BH) plus access point (AP) nodes interconnected to form a wireless mesh, which provides multi-hop paths to/from terminal stations (STAs) to a wired core infrastructure. Note that, due to the nature of outdoor small cell deployments, wired connectivity is only reached through a limited set of nodes, acting as gateways (GW). The scheme representing this architecture is depicted in Fig. 1.

In that figure, we can see how unified "access/backhaul" network elements are composed of hardware boxes containing multiple Wi-Fi interfaces, each one being used either for the access or backhaul network. AP interfaces are controlled by a logically centralized access controller (ANC), and backhaul bound interfaces are controlled by a central backhaul controller (BHC). In addition, a logical interface between the access and 


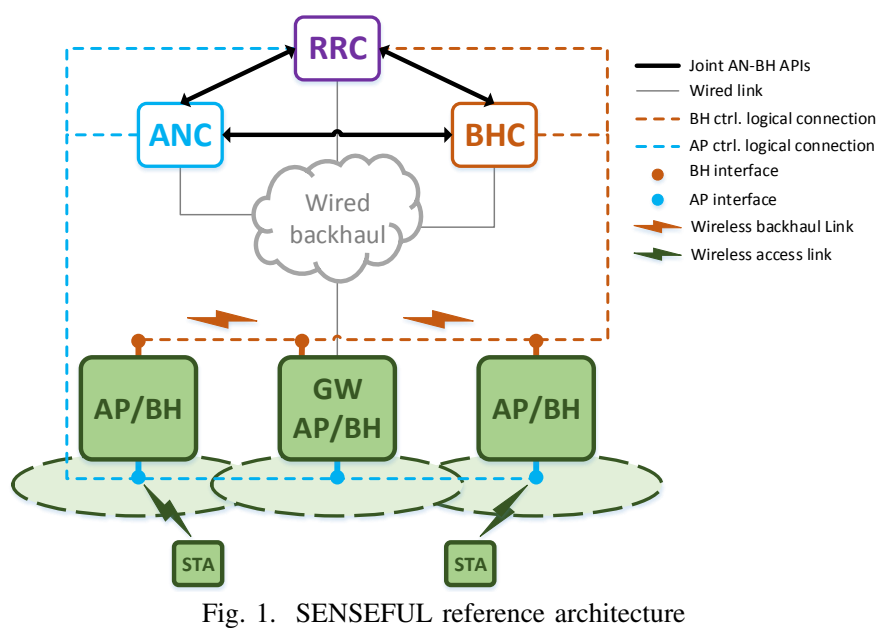

the backhaul controllers is required to enable coordination between both. A third logical entity, the radio resource controller (RRC) distributes resources between both segments. Notice that the proposed architecture allows for: i) multi-tenancy where, in general, access and backhaul could be managed by different network operators; ii) for an independent evolution of the technologies employed in the access and backhaul; iii) for control plane scalability, since access and backhaul functions are decoupled; and iv) for joint access backhaul resource management functions such as load balancing.

As detailed in the following sections, the access controller is based on BigAP [4], and the backhaul control plane follows the architecture proposed in [5].

\section{A. Backhaul control}

The proposed technology allows a transport node equipped with multiple wireless interfaces (i.e. IEEE 802.11-based) to be managed by an SDN controller. In particular, the wireless links connecting to neighbouring backhaul nodes are abstracted inside the transport node as virtual point-to-point interfaces, which appear to the SDN controller as Ethernet ports connecting those wireless switches. Thus, the SDN controller can control forwarding across the wireless backhaul in the same way as in a traditional wired network. In addition, this technology allows the transport switch to obtain, for each wireless port, real time measurements about the wireless channel, which are then reported to the SDN controller. Hence, this architecture enables different centralized radio resource management strategies. In our implementation, this ability is used to minimize interference; more precisely, the path selected for a new flow is the one that minimizes the maximum channel utilization observed by any backhaul node.

Figure 2 illustrates the architecture of a wireless backhaul node, where we can observe the following major components: i) One or more wireless devices $(D)$ controlling access to the wireless medium; ii) One $\operatorname{SDN}$ agent $(A)$ controlling the forwarding plane in the wireless switch and communicating with the remote SDN controller $(C)$, through the OpenFLowbased $\operatorname{Ext}_{S B}$ interface; and iii) A functional entity $(M U X)$, which multiplexes multiple wireless links over a single wire-

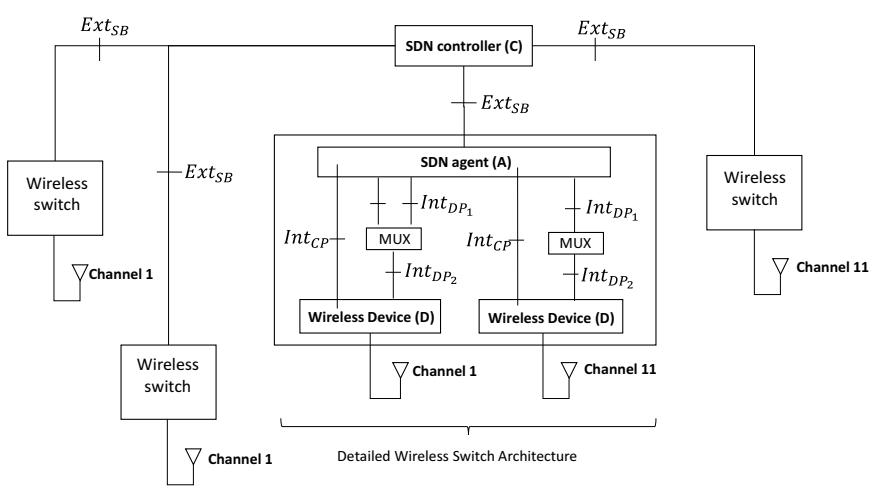

Fig. 2. Architecture of SENSEFUL's SDN wireless backhaul node [5]

less device $D$. The interfaces $I n t_{D P i}$ and $I n t_{C P}$ are used to transport data plane and control plane packets, respectively.

It is worth noting that the $M U X$ is a functional entity in charge of multiplexing data packets coming from the SDN Agent's ports, and de-multiplexing them in the reverse direction. Due to its widespread support, we use IEEE 802.1Q VLANs as a multiplexing mechanism. In particular, upon receiving a packet from $A$, the $M U X$ pushes the $\operatorname{VLAN}_{i}$ tag to the packet and forwards the packet through the wireless interface. On the other hand, upon receiving a packet from the wireless interface, the $M U X$ pops the packet's $\operatorname{VLAN}_{i}$ tag and forwards it to the appropriate interface in $A$. The interested reader is referred to [5] for a comprehensive description of this architecture and for a detailed explanation about the agreement of VLAN tags among wireless nodes.

As shown in Fig. 1, some SENSEFUL nodes have colocated access and backhaul interfaces. This requires a nontrivial adaptation of the architecture depicted in Fig. 2, since AP interfaces must be added to the SDN switch $(A)$ so that packets from/to associated Wi-Fi STAs are properly handled.

\section{B. Access control}

The wireless access network is based on the BigAP approach [4], [6], a centralized architecture for enterprise WiFi networks providing support for seamless handover, for mobility management and load balancing. BigAP does not require any hardware/driver changes on the client or AP side and is therefore fully compatible with commodity $802.11 \mathrm{n} / \mathrm{ac}$ cards supporting Dynamic Frequency Selection (DFS). BigAP decides on the channel assignment to APs on a long-term basis whereas the decision by which AP a particular STA is served is based on short-term information like channel-state information (mobility) and traffic conditions (load balancing). One of the key features of the BigAP approach is its seamless handover, which can be performed with nearly zero network-outage. Current state-of-the-art approaches for transparent networkdriven handover (i.e. without modifications on client device) are all based on the DenseAP hard-handover scheme [7], which causes a huge network outage approximately 32 times higher than the BigAP [4]. This is caused by the amount of time the STA needs for the connection build-up with the new AP, e.g. scanning/probing, authentication and re-association. BigAP, in contrast, removes all aforementioned delays by transferring 


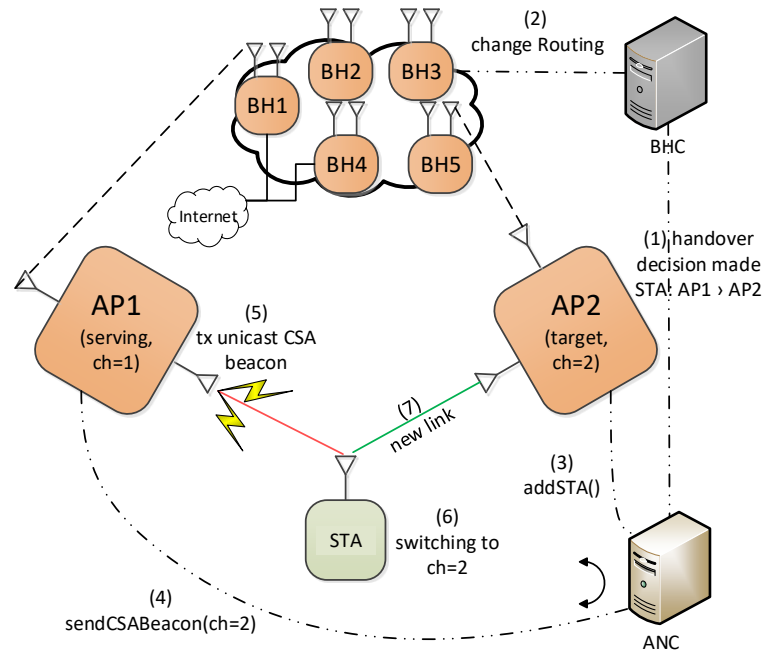

Fig. 3. (1) Decision to handover STA from $A P 1$ to $A P 2$. (2) Flows towards $S T A$ need to be routed via $A P 2, \mathrm{BHC}$ has to reconfigure routing. (3) ANC associates and authenticates $S T A$ on $A P 2$. (4-5) ANC instructs $A P 1$ to send unicast beacon containing CSA-IE with channel of AP2. (6) Receiving the beacon, STA switches the channel of AP2 (7) As both APs have the same BSSID, STA does not notice that it is being served by another AP, STA continues with its communication.

the current state of the STA from the serving AP to the target AP before the actual handover is triggered. To enable this possibility, the BigAP topology uses a single global BSSID for all APs. From the STAs point of view, the whole ESS seems like one BSS or one big AP.

To reduce packet collisions and duplicate packets (due to the use of same BSSID) and to enable spatial reuse, BigAP uses different RF channels for co-located APs. For the handover process, BigAP exploits the IEEE 802.11 DFS functionality and leads the STA to believe that the serving AP will perform a RF channel switch while, actually, the serving AP remains on its current RF channel but the target AP is operating on the new RF channel. In consequence, the STA believes it is still associated to the same AP, although it is not. By relying on these principles the communication can be continued without any further outage except for the time needed to switch the channel in the STA.

BigAP exploits the possibility of DFS to announce channel switches to trigger a channel switch within STAs and further to perform the handover operation. To achieve this, the centralized BigAP access network controller (ANC) instructs the serving AP to send a unicast beacon frame containing a Channel Switch Announcement Information Element (CSAIE) with the RF channel of the target AP. Reception of CSAIE triggers the channel switching in the STA to the desired RF channel. As an illustrative example Fig. 3 shows the required steps to perform a handover of STA from AP1 to AP2.

\section{Joint access-backhaul control}

In order to validate the architecture and illustrate the benefits of joint access/backhaul coordination, two use cases are implemented and demonstrated: i) backhaul-aware access network control and ii) access/backhaul resource management.
1) Backhaul-aware access network control: the status of the wireless backhaul impacts the quality of service offered to the clients served through the access network. Therefore, the new access network control is able to provide association/handover decisions based on the status of the backhaul. On the other hand, network controlled association and handover entails creation/modification of optimal backhaul paths, as depicted in Fig. 3. To ease the optimization of the backhaul, the ANC informs the BHC of association/handover decisions. In SENSEFUL, this is achieved by means of a REST API between the controllers:

- $\mathrm{ANC} \rightarrow$ BHC: POST new client station (STA) to inform upon a new association; the backhaul then computes the best path from the selected access point (AP) to the core gateway. The response to this call is a metric that corresponds to the backhaul capacity available through that AP; therefore, this call can be also used to decide the best candidate AP for a given STA, based on the status of the backhaul.

- $\mathrm{ANC} \rightarrow \mathrm{BHC}$ : PUT new station association information to inform of a network-driven handover (see previous bullet point).

- $\mathrm{ANC} \rightarrow \mathrm{BHC}:$ DELETE station when it leaves the access network; the backhaul controller removes the corresponding forwarding rules.

- $\mathrm{BHC} \rightarrow \mathrm{ANC}$ : POST a suggested handover in order for the backhaul controller to notify congestion. The call also suggests a possible handover that is expected to alleviate congestion in the backhaul.

2) Access/Backhaul resource management: the scarce radio resources available in the unlicensed bands used by SENSEFUL Wi-Fi access and backhaul networks require an intelligent management. Note that the CSMA used in $\mathrm{Wi}-\mathrm{Fi}$ will provide equal access probability to $\mathrm{AP}$ and $\mathrm{BH}$ interfaces sharing the channel, when the latter has to transport more traffic/flows (aggregation of multiple access links) and, therefore, deserves more resources. Thus, centralized scheduling of AP and $\mathrm{BH}$ interfaces using a hybrid TDMA/CSMA scheme provides enhanced flexibility by allowing fair distribution of resources between both networks and reduces collisions inherent to WiFi. What is more, that flexibility brought by TDMA would enable the application of advanced network functions such as network slicing [8]. hMAC [9] is a technique to provide a TDMA-like access in Wi-Fi interfaces. hMAC's current implementation $^{1}$ is based on the idea of using a hybrid TDMA/CSMA control protocol, where local or remote (i.e. RRC) scheduling of the different IEEE 802.11's EDCA queues at the driver level (ath9k), control outgoing flows. Later, the "real" over-the-air transmission is achieved by following the default IEEE 802.11 CSMA/CA rules. Time slots available at each radio link are thus managed by the RRC, which actually resides in the access controller. The RRC then communicates the scheduling to the access points $\left(\mathrm{AP}_{x}\right)$ and backhaul nodes $\left(\mathrm{BH}_{y}\right)$ using an agent deployed at each device. Figure 4

\footnotetext{
${ }^{1}$ https://github.com/szehl/ath9k-hmac
} 


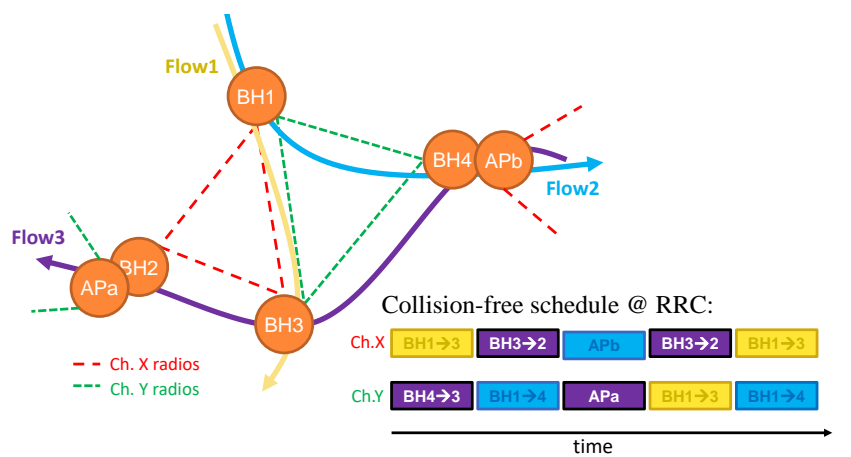

Fig. 4. Example of scheduling of a TDMA-based backhaul and access network infrastructure

provides an example of such hybrid TDMA scheduling, where four $\mathrm{BH}$ nodes and two AP nodes share two radio channels (Ch. X and Ch. Y) to serve three data flows.

\section{TESTBED DESCRIPTION}

In order to evaluate a proof-of-concept of the SENSEFUL architecture, a particular topology was designed, which is depicted in Fig. 5. The scenario depicted in Fig. 5 was implemented and validated in the TWIST testbed [10] in Technical University Berlin (TUB). These are the different elements represented in the figure.

- STA: mobile terminals equipped with Wi-Fi (5xTP-Link WDR4300 with OpenWRT and 1x Android smartphone)

- $B H$ : wireless backhaul nodes (Intel NUC) with customized Linux (including BigAP and SDN agents).

- $A P / B H$ : nodes operating both as access (AP) and backhaul $(\mathrm{BH})$ nodes.

- $B H / G W$ : endpoint of wireless backhaul.

- CORE network: access network controller, backhaul controller (with OpenDaylight) and traffic source server.

IP addressing is divided into three domains: i) access network, including end user stations (STAs) and AP interfaces; ii) backhaul network; and iii) core network. In the experiment, all STAs except for STA2 are static and act as traffic sinks (traffic used to load the network is generated from the core). Note that one of the key features of BigAP is that it is transparent to legacy Wi-Fi STAs in order to keep backwards compatibility and, therefore, hMAC is not implemented in those STAs (i.e. TDMA not available in the uplink); hence the evaluation in the downlink only. STA2, on the other hand, supports the networkdirected handover managed by the BigAP framework; that is, STA2 must support IEEE 802.11h.

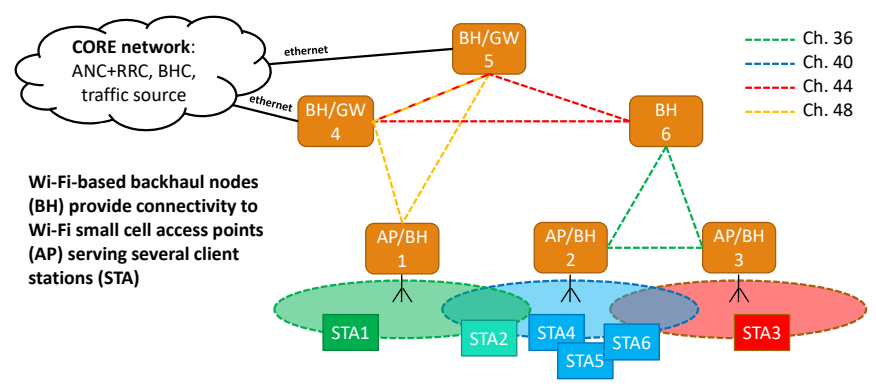

Fig. 5. topology of the SENSEFUL experiment
Each BH device is equipped with two Atheros-based IEEE 802.11abgn interfaces operating in the $5 \mathrm{GHz}$ band (see channel distribution in Fig. 5). In the case of AP/BH nodes, one of the interfaces is used to serve as an AP to provide access to STAs, and the other one is used to join the wireless backhaul. Therefore, the three AP/BH nodes participate both in the access network (as part of the BigAP) and the backhaul (managed from an external SDN controller). There is a pure $\mathrm{BH}$ node, which uses its two radio interfaces to participate in the backhaul infrastructure as a wireless switch. The two $\mathrm{BH} / \mathrm{GW}$ nodes are gateway nodes that connect the wireless infrastructure to the wired core through an Ethernet connection. We have to note that the nodes are distributed in different rooms; in order to minimize the differences between LoS and non-LoS links and for the sake of reproducibility, we limit all the Wi-Fi links to the most robust modulation (6 Mbps). With such configuration, all nodes are within reach of each other; then, we emulate the limited coverage of an AP by blacklisting the STAs it will not serve.

Backhaul control is implemented in an OpenDaylight (ODL) platform and runs in a remote location (accessed through a VPN from GW nodes). Access control, on the other hand, is allocated in a server with direct access to the testbed (direct link to $\mathrm{BH} / \mathrm{GW}$ nodes). Note that, for this experiment, the radio slot control (TDMA scheduling) also resides in the access server. In this implementation, the access controller provides a scheduling based on the number of flows going through each link $^{2}$, information that can be provided by the backhaul controller. A third server is used as TCP traffic source, where downlink traffic is generated with iperf tool.

Finally, we have to note that the deployment of a dynamic TDMA scheduling by the controller, as described in section II-C, requires a precise synchronization of the nodes so that the time slots defined start at precisely the same instant in all the devices sharing the medium. In this work, synchronization is provided through a common Ethernet infrastructure. However, in a real deployment, such infrastructure will not be present and synchronization is provided over the air. This poses other interesting challenges, out of the scope of this project. Using IEEE 1588 PTP (Precision Time Protocol) we measured, on average, $50 \mu$ s of synchronization error, where $99 \%$ of the time that error was below $1 \mathrm{~ms}$ and below $100 \mu \mathrm{s}$ for more than $91 \%$ of the time samples. PTP thus provides enough precision for the time scales of the hMAC, where slot durations range from 1 to $20 \mathrm{~ms}$.

\section{Measurement-BASED EVAluation}

The scenario depicted in Fig. 5 was set up in order to assess the potential of the joint operation of the access network controller (ANC) and the backhaul controller (BHC). In that scenario, we run different sets of experiments. The first

\footnotetext{
${ }^{2}$ We assume all flows are equal and that all links have similar characteristics, therefore, the number of flows constitutes a good metric to compute fair slot allocations. In future developments, the scheduling algorithm could be further sophisticated to consider different flows, with different QoS requirements, and radio links with different performance metrics.
} 


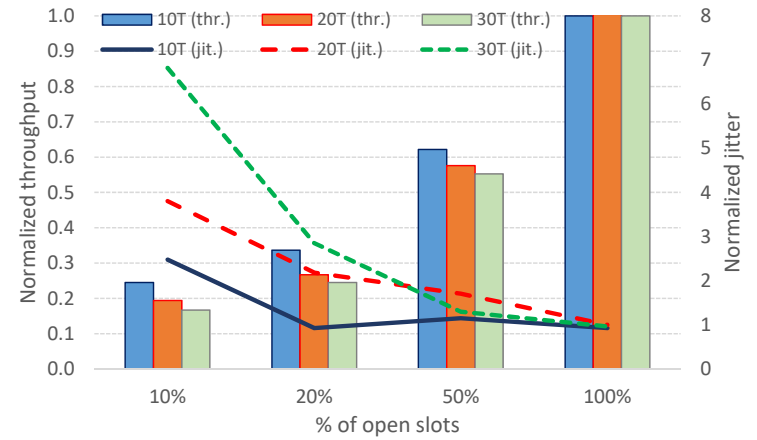

Fig. 6. Normalized (wrt CSMA) throughput and jitter measurements of hMAC with different portion of available slots, and different slot duration (in terms of frame transmission time $T$ )

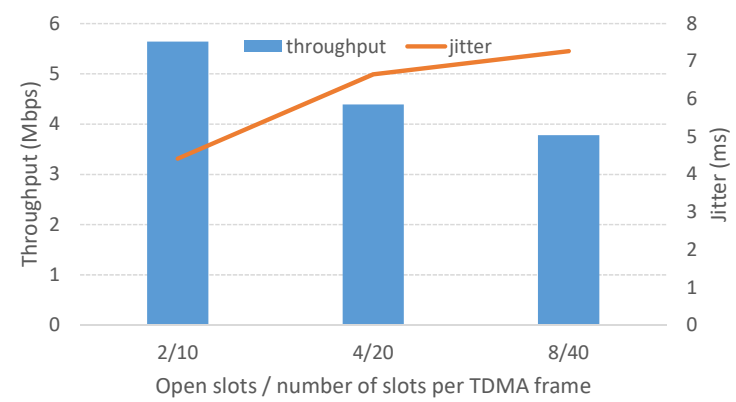

Fig. 7. Throughput and jitter of hMAC with different TDMA frame sizes and constant proportion of open slots

one was intended to adjust the configuration of the hMAC parameters and the second is used to highlight the benefits of the SENSEFUL architecture.

\section{A. Tuning of hybrid CSMA/TDMA parameters}

In this phase, optimal TDMA frame and slot sizes were selected for the rest of the experiments. Due to limitations of current hMAC implementation [9], the slot duration should allow for, at least, ten transmissions (up to $20 \mathrm{~ms}$, when lowest rates are used). However, performance of hMAC's TDMA transmissions perform worse as the slot size is increased. Figure 6 shows throughput (bars) and jitter (lines), normalized to the values measured for legacy CSMA/CA, achieved with different slot durations (in terms of the average frame transmission time $T$ ). Similarly, we found that the TDMA frame should be kept small as well. Figure 7 shows throughput (bars) and jitter (line) measured for different sizes of the TDMA frame. Note that the portion of used slots with respect to the TDMA frame is always the same (i.e. 20\%) and, therefore, the resulting throughput should be the same. However, longer TDMA frames entail larger time between consecutive available slots, thus increasing delay and jitter, which may incur in retransmissions and a loss of efficiency.

\section{B. SENSEFUL in action}

In a second set, we compare two different cases: Legacy, where there is no cooperation between access and backhaul control and medium access follows the legacy distributed CSMA/CA of IEEE 802.11; and SENSEFUL, implementing the proposed joint control of access and backhaul. Those two experiments follow a particular sequence, shaping a "story" that highlights the benefits of the proposed scheme.

During the initialization phase of the experiment, the topology is built and the BHC starts receiving reports from the nodes (i.e. radio and packet statistics and list of neighbors). The experiment then follows the particular sequence detailed below. Fig. 8 shows the evolution of aggregated throughput and fairness throughout that sequence for the two cases: Legacy and SENSEFUL. Fairness is computed on a per-flow basis following Jain's formulation [11]. Note that whenever a new STA is associated, a new downlink TCP flow is initiated in the core towards that STA. Also recall that all radio links were limited to $6 \mathrm{Mbps}$.

1) STA1 is switched on (only reaches AP1):

a) ANC handles association of STA1 with AP1 and notifies the backhaul controller using the API described in II-C.

b) BHC computes the optimal path between the core and STA1 and sets new forwarding rules on the corresponding backhaul nodes (BH1, and BH4).

After this first step, STA1 achieves around 2.7 Mbps.

2) STA2 switched on (can reach AP1 and AP2):

a) ANC decides association of STA2 to AP2 and notifies the backhaul controller.

b) BHC computes the optimal path between the core and STA2 and sets new forwarding rules on the corresponding nodes (BH2, BH6 and BH5).

In this step, STA1's throughput is reduced to $1.7 \mathrm{Mbps}$ and STA2 achieves $0.9 \mathrm{Mbps}$. Note that both AP1 and BH6 compete for the same channel 36. We also have to highlight here that the link $\mathrm{BH} 5 \rightarrow \mathrm{BH} 6 \rightarrow \mathrm{BH} 2$ has a capacity of only $1.5 \mathrm{Mbps}^{3}$. The differences in throughput decreases fairness.

3) STA3 switched on (only reaches AP3):

a) ANC handles association of STA3 with AP3 and notifies the backhaul controller.

b) BHC computes the optimal path between core network and STA3 and sets new forwarding rules on the corresponding nodes (BH3, BH6 and BH5).

c) Given that now BH6 carries two flows through channel 36, while AP1 carries only one, the SENSEFUL approach activates hMAC to produce a fairer share of channel 36 (and channel 44, shared between BH5 and AP3).

SENSEFUL approach achieves near-perfect fairness while, in the Legacy network, this parameter degrades. On the other hand, throughput is notably reduced after applying hMAC.

4) STA4, 5 and 6 are switched on (only reach AP2):

a) ANC handles association of those STAs with AP2 and notifies the backhaul controller.

b) BHC computes the optimal path between core network and STAs 4, 5 and 6 and sets new forwarding rules on the corresponding backhaul nodes $(\mathrm{BH} 2$, BH6 and BH5).

${ }^{3}$ Probably due to internal interference between co-located radios in BH6 


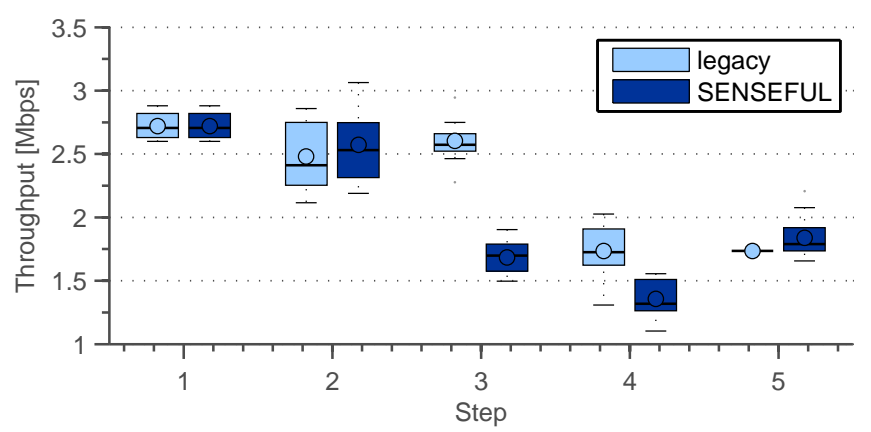

(a) Throughput

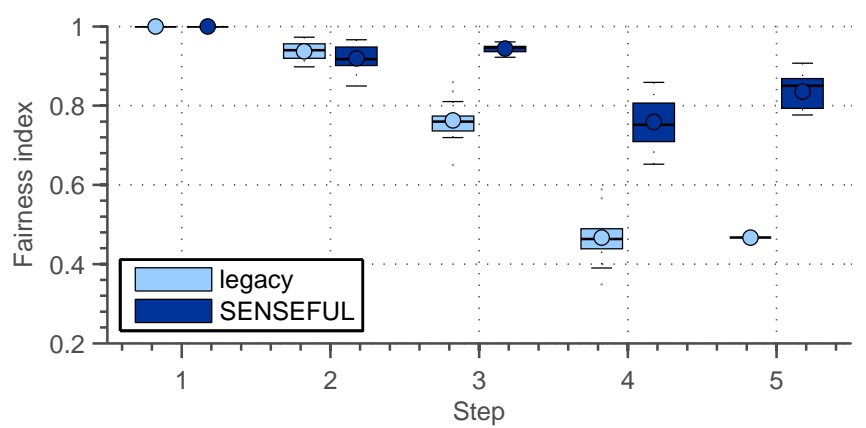

(b) Fairness

Fig. 8. Throughput and fairness measurements for SENSEFUL and Legacy approaches for the five steps. Shown are quartiles, median and mean (circle)

c) According to the new balance of data flows, SENSEFUL determines a new TDMA scheduling.

Due to the increased interference, in the Legacy case both throughput and fairness are notably reduced while SENSEFUL is capable of keeping a reasonably high fairness without losing capacity with respect to the previous step.

5) SENSEFUL requests a handover:

a) BHC detects congestion (i.e. measured channel utilization exceeds a configurable threshold) and suggests the ANC to move STA2 from AP2 to AP1 using the API described in II-C.

b) In SENSEFUL, the ANC proceeds with the handover of STA2 as suggested, then it informs the $\mathrm{BHC}$ through the API and, hence, BHC reconfigures the layer 2 path for STA2's flows accordingly (i.e. STA2 does not change its IP address). The handover process is exemplified in Fig. 3.

c) The change in the balance of flows triggers a new TDMA slot allocation.

Upon detecting congestion, the BHC looks for STAs under the coverage of multiple APs (data updated by the ANC) and makes an estimation of the new utilization of the most congested backhaul link after a possible handover. If that utilization improves the current state by a given threshold, the handover is suggested to the ANC through the API.

Note that this step does not occur in the Legacy case (in step 5, Fig. 8 shows the average value measured during step 4 for the Legacy case); even though the ANC (based on BigAP) alone could manage association of STAs, note that AP2 is not seen as congested (channel 40 is clean and AP2 carries less than $700 \mathrm{kbps}$ ) and, hence, it would not trigger a reassociation. The SENSEFUL approach is capable of reducing the congestion thus improving fairness and throughput.

\section{CONCLUSIONS}

In dense scenarios, joint control of access and backhaul allows the implementation of new strategies (e.g. backhaulaware handovers or centralized TDMA scheduling) with evident benefits over a network where access and backhaul operate independently. Benefits are seen even in the relatively simple scenario studied in this work, but we would like to highlight that the advantages of the coordinated access/backhaul are not only measured with conventional KPIs (fairness, throughput) but also in intangible terms such as flexibility.

Network-driven association control helps in reducing congestion in backhaul links and, at the same time, backhaul control helps in achieving better association management, benefiting the end user. Furthermore, the use of a TDMAlike access brings additional benefits by providing improved fairness and, more interestingly, its flexibility enables the operator to establish different policies to create and differentiate services or even to instantiate multiple network slices. The use of hMAC, however, has caveats since its application could impact throughput negatively. Hybrid TDMA/CSMA brings evident benefits in a congested channel; without congestion, Wi-Fi's legacy CSMA proved to be more efficient.

\section{ACKNOWLEDGMENT}

The research leading to these results has received funding from the European Union under grant agreements 671551 (H2020 5G-XHaul) and 645274 (H2020 WiSHFUL) and from the Spanish government through TEC2016-76795-C6-2-R.

\section{REFERENCES}

[1] "Cisco Visual Networking Index: Global Mobile Data Traffic Forecast Update 2015-2020," Cisco Systems, Inc., Tech. Rep., February 2016.

[2] (2017) Small Cell Forum website. [Online]. Available: http://www. smallcellforum.org

[3] O. Aboul-Magd, "The Future of Wi-Fi Technology: Looking Ahead to the Future," in HENC 2013, 2013.

[4] A. Zubow, S. Zehl, and A. Wolisz, "BIG AP - Seamless Handover in High Performance Enterprise IEEE 802.11 Networks," in 15th IEEE/IFIP IEEE NOMS 2016, 2016.

[5] A. Hurtado-Borràs, J. Palà-Solé, D. Camps-Mur, and S. Sallent-Ribes, "SDN wireless backhauling for Small Cells," in IEEE International Conference on Communications (ICC), June 2015.

[6] S. Zehl, A. Zubow, and A. Wolisz, "BIG AP - A Seamless Handover Scheme for High Performance Enterprise IEEE 802.11 Networks," in 15th IEEE/IFIP IEEE NOMS 2016, April 2016.

[7] R. Murty, J. Padhye, R. Chandra, A. Wolman, and B. Zill, "Designing high performance enterprise wi-fi networks." in NSDI, vol. 8, 2008.

[8] S. Zehl, A. Zubow, and A. Wolisz, "Hotspot slicer: Slicing virtualized home Wi-Fi networks for Air-Time guarantee and traffic isolation," in IEEE 18th WoWMoM 2017, Macau, P.R. China, Jun. 2017.

[9] S. Zehl, A. Zubow, and A. Wolisz, "hMAC: Enabling Hybrid TDMA/CSMA on IEEE 802.11 Hardware," arXiv:1611.05376, 2016.

[10] TWIST, “Tkn wireless networks testbed," https://www.twist.tu-berlin $\mathrm{de} /$, (Accessed on 01/26/2017).

[11] R. Jain, D. Chiu, and W. Hawe, "A quantitative measure of fairness and discrimination for resource allocation in shared computer systems,' DEC Research, Tech. Rep., September 1984. 